\section{AMINOGLYCOSIDE-INDUCED MODIFICATIONS OF MEMBRANE PHOSPHOINOSITIDE METABOLISM. A COMPARATIVE STUDY}

\author{
Pierre Marche* and Arlette Girard \\ Inserm U7, Hopital Necker, \\ Département de Néphrologie, \\ 161 Rue de Sèvres, 75015 Paris, France
}

(Received for publication January 24, 1984)

Several lines of evidence indicate that the first steps in the pathogenesis of aminoglycoside nephrotoxicity involve the accumulation of the antibiotic in the proximal tubule where it interacts with the plasma membrane of epithelial cells ${ }^{1,2)}$. In brush border membranes, acidic phospholipids and most particularly phosphoinositides, phosphatidylinositol (PI), phosphatidylinositol-4phosphate (PI-P) and phosphatidylinositol-4,5bisphosphate (PI- $\mathrm{P}_{2}$ ), have been recently shown to be an integral component of the aminoglycoside binding site $^{3)}$. In addition, the aminoglycosides have been shown to modify the metabolism of phosphoinositides in the kidney cortex ${ }^{4 \sim 7)}$. In a biomembrane model such as the ghost membrane isolated from rat erythrocyte, results from this laboratory ${ }^{8)}$ indicated that aminoglycosides impaired the phosphoinositide interconversion since these drugs induced a decrease and an increase in the ${ }^{52} \mathrm{P}$-labelling of PI-P $\mathrm{P}_{2}$ and PI-P, respectively likely by inhibiting the PI-P kinase; the existence of a possible relationship between the toxicity of aminoglycosides and their capacity to impair the phosphoinositide metabolism was also suggested. This finding stimulated us to further study the interaction between aminoglycosides and phosphoinositide metabolism within the membrane. For this purpose, using isolated red cell ghosts, experiments were designed to compare the effects of five aminoglycosides in clinical use, namely amikacin, dibekacin, gentamicin, netilmicin and tobramycin on the ${ }^{32} \mathrm{P}$ labelling of the polyphosphoinositides PI-P and PI-P .

\section{Materials and Methods}

Fifteen to eighteen week-old male rats of the Wistar strain were supplied by Iffa Credo
(France). $\quad\left[\gamma^{-32}\right.$ P]ATP $(2,000 \sim 3,000 \mathrm{Ci} / \mathrm{mmol})$ was purchased from Amersham (UK). Amikacin, dibekacin and netilmicin were generously provided by Bristol, Bristol/Bellon and Unilabo, respectively. Gentamicin and tobramycin were from Sigma. Blood sampling and preparation of isolated ghost membranes were performed as already detailed ${ }^{9)}$. For ${ }^{32} \mathrm{P}$ incorporation into membrane phospholipids, $0.3 \mathrm{mg}$ membrane protein were preincubated for 10 minutes at $37^{\circ} \mathrm{C}$ in $50 \mathrm{~mm}$ Tris- $\mathrm{HCl}$ buffer $\mathrm{pH} 7.5$ in the presence (or absence for controls) of the antibiotic. Then $5 \mathrm{mM} \mathrm{MgCl}_{2}$ and $2 \mathrm{mM} \mathrm{ATPNa}_{2}$ (containing $5 \sim$ $\left.10 \mu \mathrm{Ci}\left[\gamma_{-}{ }^{32} \mathrm{P}\right] \mathrm{ATP}\right)$ final concentrations, were added in a total volume of $0.5 \mathrm{ml}$. The reaction was stopped 15 minutes later and samples were processed for phospholipid extraction, separation and ${ }^{32} \mathrm{P}$-phosphoinositide analysis as described previously ${ }^{9}$. In order to compare the effects of antibiotics on the ${ }^{32} \mathrm{P}$ incorporation into phosphoinositides, the following experimental protocol was used. In a single experiment, the five aminoglycosides namely, amikacin, dibekacin, gentamicin, netilmicin and tobramycin were studied on the same membrane preparation obtained from one rat. Each aminoglycoside was studied in duplicate at two concentrations $(6 \times$ $10^{-5}$ and $6 \times 10^{-4} \mathrm{M}$ ). Control samples (processed in the absence of drug) were in quadruplicate. The ${ }^{32} \mathrm{P}$ radioactivities associated with PI- $P_{2}$ and PI-P in each drug-treated sample were expressed as the percentage of these radioactivities in controls. Seven experiments were performed, each one using a different membrane preparation in order to take into account variations between animals.

\section{Results and Discussion}

Using isolated ghosts as a biomembrane model, a previous study has shown that concentration of $6 \times 10^{-5} \mathrm{M}$ neomycin was required to induce an increase in $\left.{ }^{32} \mathrm{P}\right] \mathrm{PI}-\mathrm{P}$ as well as a concomitant decrease in $\left.{ }^{32} \mathrm{P}\right] \mathrm{PI}-\mathrm{P}_{2}$. We therefore chose aminoglycoside concentrations of $6 \times 10^{-5}$ and $6 \times 10^{-4} \mathrm{M}$ for the present study. When used at $6 \times 10^{-5} \mathrm{M}$, all the drugs tested did not significantly affect the ${ }^{32}$ P-labelling of PI-P $_{2}$ (Fig. 1). At such a concentration, whereas amikacin did not modify significantly the ${ }^{32} \mathrm{P}$-labelling of PI-P this latter was significantly enhanced by the other aminoglycosides studied (Fig. 1). When used at 
Fig. 1. Effect of aminoglycosides on the ${ }^{32}$ P-labelling of PI-P $P_{2}$ (upper) and of PI-P (lower).

Values are expressed as the percentage of those obtained in controls (i.e. when the drugs were omitted) which were $1.67 \pm 0.19$ and $0.60 \pm 0.08{ }^{32} \mathrm{P}$ $\mathrm{nmol} / 15$ minutes/mg protein for $\left[{ }^{32} \mathrm{P}\right] \mathrm{PI}-\mathrm{P}_{2}$ and $\left[{ }^{32} \mathrm{P}\right] \mathrm{PI}-\mathrm{P}$, respectively (mean $\pm \mathrm{SEM}, \mathrm{n}=7$ ).

Gentamicin, dibekacin, $\triangle$ netilmicin, $\Delta$ amikacin, $\square$ tobramycin.
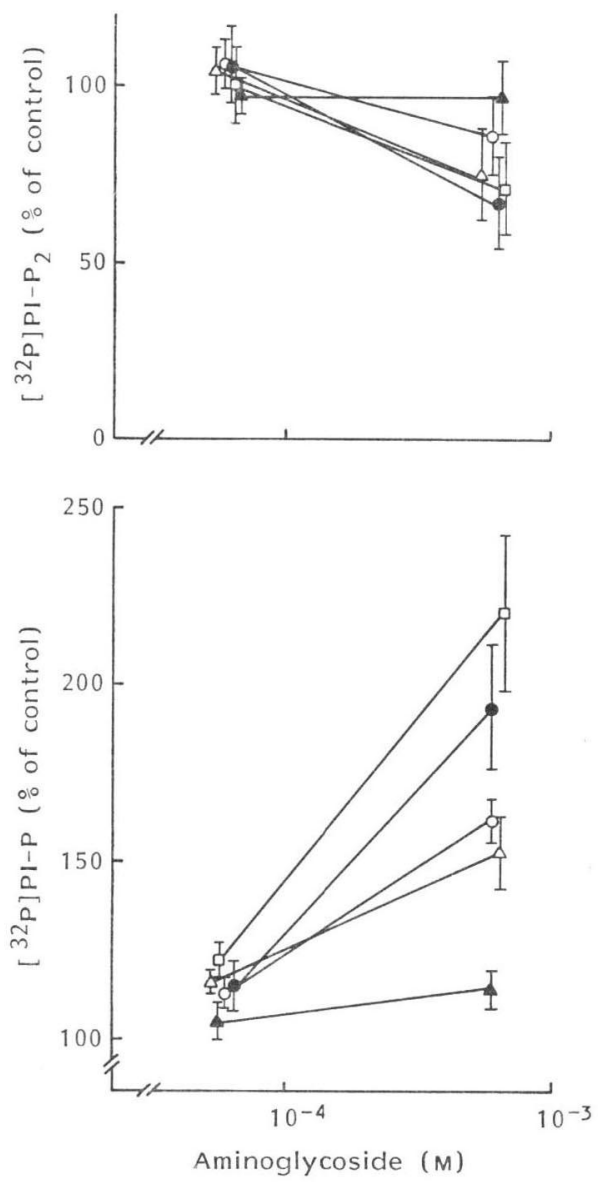

$6 \times 10^{-4} \mathrm{M}$, Fig. 1 also shows that all aminoglycosides tested, except amikacin, induced a significant decrease in $\left[{ }^{32} \mathrm{P}\right] \mathrm{PI}-\mathrm{P}_{2}$ and a significant increase in $\left[{ }^{32} \mathrm{P}\right]$ PI-P. It is noteworthy that the total radioactivity incorporated into both PI-P ${ }_{2}$ and PI-P in aminoglycoside-treated samples (and irrespective of the drug used) did not differ significantly from that incorporated into controls $(2.16 \sim 2.51$ versus $2.27{ }^{32} \mathrm{P}$ nmol/15 minutes/mg protein). This agrees with what has been reported with neomycin-treated samples ${ }^{8)}$ and suggests that aminoglycosides share a similar mechanism of action for impairing the phosphoinositide interconversion.

Although the potency with which a single aminoglycoside affected the ${ }^{32} \mathrm{P}$ incorporation into PI-P and PI-P 2 , varied with the animal used for membrane preparation, the data obtained in the course of our experiments revealed that the order of potency of the drugs studied was very similar from one experiment to another. This prompted us to use the paired Student's t test for comparing the five aminoglycosides studied. When the antibiotics were used at $6 \times 10^{-5} \mathrm{M}$, no significant difference could be detected between them, probably because at this concentration the drug-induced modifications were too small (Fig. 1). When the drugs were used at $6 \times 10^{-4} \mathrm{M}$ the statistical analysis revealed that: if one considers their ability to decrease $\left[{ }^{32} \mathrm{P}\right] \mathrm{PI}-\mathrm{P}_{2}$, (i) amikacin was less effective than dibekacin $(P<$ 0.05), netilmicin $(P<0.02)$, gentamicin and tobramycin $(P<0.001)$ and (ii) gentamicin was less effective than dibekacin $(P<0.05)$ and than tobramycin $(P<0.01)$; if one considers their ability to increase [ $\left.{ }^{32} \mathrm{P}\right] \mathrm{PI}-\mathrm{P}$, (i) amikacin was less effective than the other four antibiotics $(P<0.01$ vs. dibekacin, tobramycin or netilmicin and $P<$ 0.001 vs. gentamicin), (ii) netilmicin, gentamicin and dibekacin were less effective than tobramycin $(P<0.01)$ and (iii) netilmicin was less effective than dibekacin $(P<0.01)$. Not included in this comparative investigation, a separate experiment showed that neomycin was more potent than tobramycin. Indeed when compared to control values, $\left[{ }^{32} \mathrm{P}\right] \mathrm{PI}-\mathrm{P}_{2}$ and $\left[{ }^{32} \mathrm{P}\right] \mathrm{PI}-\mathrm{P}$ were 51 and $222 \%$, respectively in the presence of $6 \times 10^{-4} \mathrm{M}$ tobramycin whereas these values were 28 and $323 \%$, respectively in the presence of $3 \times 10^{-4} \mathrm{M}$ neomycin.

Aminoglycoside concentrations in the millimolar range could not be used appropriately in the system studied since too high drug concentrations have been proved to decrease both [ $\left.{ }^{32} \mathrm{P}\right] \mathrm{PI}-\mathrm{P}_{2}$ and $\left[{ }^{32} \mathrm{P}\right] \mathrm{PI}-\mathrm{P}$ (results not shown). Although the use of only two drug concentrations prevents calculation of $\mathrm{ED}_{50}$ values for the compounds tested, the experimental design of the present investigation nevertheless allows the comparative study of the effect of aminoglycosides on the phosphoinositide interconversion. The results clearly indicate that tobramycin is 2 times as potent as amikacin. On the other hand, the results do not permit a clear distinction between dibekacin, genta- 
micin and netilmicin which were approximately 1.4 1.7 times as potent as amikacin. Thus the order of ability to impair the ${ }^{32} \mathrm{P}$-labelling of polyphosphoinositides is: tobramycin $>$ gentamicin $\simeq$ dibekacin $\simeq$ netilmicin $>$ amikacin; neomycin appears to be more potent than tobramycin. In addition, streptomycin has been described as similar to amikacin ${ }^{8)}$. The above ranking order resembles the order of aminoglycosides when ranked according to their cationic charge (streptomy$\operatorname{cin}=3$; amikacin $=4$; tobramycin $=$ netilmicin $=$ dibekacin $=$ gentamicin $=5$; neomycin $=6$ ). This suggests that a charge interaction between the basic drugs and the acidic membrane phospholipids could account, at least in part, for the effects observed here. Such an interaction has been described to be involved in the binding of aminoglycosides to brush border membranes ${ }^{32}$. However, our observation that tobramycin was significantly more potent than dibekacin, gentamicin and netilmicin suggests also that other determinants such as the molecular structure may confer to an aminoglycoside its potency to impair the phosphoinositide metabolism.

The nephrotoxic potential of aminoglycosides appears also to correlate with their cationicity (for review see ref 10). This potential appears however to be determined by both the extent to which the drug accumulates within tubules and the intrinsic toxic effect of the drug on the tubular cell itself ${ }^{11)}$. In the kidney cortex, the lesser accumulation of tobramycin compared to gentamicin may explain therefore the lower degree of nephrotoxicity of the former compound ${ }^{10,11)}$ although its capacity to alter phosphoinositide metabolism was greater. The $\mathrm{Ca}^{2+}$ binding to membranes and to lipid monolayers has also been demonstrated to be altered by aminoglycosi$\operatorname{des}^{12 \sim 14)}$. Although it still remains to establish that this can be related with the drug-induced alteration of phosphoinositide metabolism, it is noteworthy that polyphosphoinositides have been described as intimately involved in the processes of transmembrane $\mathrm{Ca}^{2+}$ flux (ref 15 and references therein). These lipids also participate actively in the structure and function of membranes $^{16)}$. It is therefore conceivable that aminoglycoside-induced modifications of phosphoinositide metabolism would lead to alterations of membrane permeability and/or membrane transport capacity that can play critical roles in the pathogenesis of cellular injury. In this respect the recent identification of polyphosphoinositides and of PI-kinase activity in lysosomes ${ }^{17}$ ) may be relevant since aminoglycosides accumulate in these organelles where they cause phospholipidosis and myeloid body formation before cell necrosis.

\section{References}

1) Luft, F. C. \& S. A. Kleit: Renal parenchymal accumulation of aminoglycoside antibiotics in rats. J. Infect. Dis. 130: 656 659, 1974

2) Just, M. \& E. Habermann: The renal handling of polybasic drugs. 2. In vitro studies with brush border and lysosomal preparations. Naunyn-Schmiedeberg's Arch. Pharmacol. 300: 67 76, 1977

3) Sastrasinh, M.; T. C. Knauss, J. M. Weinberg \& H. D. HumEs: Identification of the aminoglycoside binding site in rat renal brush border membranes. J. Pharmacol. Exp. Ther. 222: 350 358, 1982

4) Feldman, S.; M.Y. Wang \& G.J. Kaloyanides: Aminoglycosides induce a phospholipidosis in the renal cortex of the rat: An early manifestation of nephrotoxicity. J. Pharmacol. Exp. Ther. 220: $514 \sim 520,1982$

5) Knauss, T. C.; J. M. Weinberg \& H. D. Humes: Alterations in renal cortical phospholipid content induced by gentamicin: Time-course, specificity and subcellular localization. Am. J. Physiol. 244 (Renal Fluid Electrolyte Physiol. 13): F535 F546, 1983

6) Lipsky, J. J. \& P. S. Lietman: Aminoglycoside inhibition of a renal phosphatidylinositol phospholipase C. J. Pharmacol. Exp. Ther. 220: 287 292, 1982

7) Schibeci, A. \& J. Schacht: Action of neomycin on the metabolism of polyphosphoinositides in the guinea pig kidney. Biochem. Pharmacol. 26: 1769 1774, 1977

8) Marche, P.; S. Koutouzov \& A. Girard: Impairment of membrane phosphoinositide metabolism by aminoglycoside antibiotics. J. Pharmacol. Exp. Ther. 227: 415 420, 1983

9) Marche, P.; S. Koutouzov \& P. Meyer: Metabolism of phosphoinositides in the rat erythrocyte membrane. Biochim. Biophys. Acta 710: 332 340, 1982

10) Humes, H. D.; J. M. Weinberg \& T. C. Knauss: Clinical and pathophysiologic aspects of aminoglycoside nephrotoxicity. Am. J. Kid. Dis. 2: $5 \sim 29,1982$

11) Soberon, L.; R. L. Bowman, E. PastorizaMunoz \& G. J. Kaloyanides: Comparative nephrotoxicities of gentamicin, netilmicin and 
tobramycin in the rat. J. Pharmacol. Exp. Ther. 210: 334 343, 1979

12) Hruska, K.A.; S.C. Mills, S. Khalifa \& M.R. Hammerman: Phosphorylation of renal brushborder membrane vesicles. J. Biol. Chem. 258: $2501 \sim 2507,1983$

13) Lodhi, S.; N. S. WeIner \& J. Schacht: Interactions of neomycin and calcium in synaptosomal membranes and polyphosphoinositide monolayers. Biochim. Biophys. Acta 426: $781 \sim 785$, 1976

14) Lüllmann, H. \& B. Vollmer: An interaction of aminoglycoside antibiotics with $\mathrm{Ca}$ binding to lipid monolayers and to biomembranes. Biochem. Pharmacol. 31: 3769 3773, 1982
15) Creba, J. A.; C. P. Downes, P. T. Hawkins, G. Brewster, R. H. Michell \& C. Kirk: Rapid breakdown of phosphatidylinositol 4-phosphate and phosphatidylinositol 4,5-bisphosphate in rat hepatocytes stimulated by vasopressin and other $\mathrm{Ca}^{2+}$-mobilizing hormones. Biochem. J. 212: $733 \sim 747,1983$

16) Mrchell, R. H.: Inositol phospholipids in membrane function. Trends Biochem. Sci. 4: 128 131, 1979

17) Collins, C. A. \& N. N. Wells: Identification of phosphatidylinositol kinase in rat liver lysosomal membranes. J. Biol. Chem. 258: 2130 2134,1983 